\title{
Uma nova metodologia para analisar raciocínios argumentativos
}

\section{A new methodology for analysing argumentative reasoning}

\author{
Marina Martins ${ }^{1}$. Rosária Justi ${ }^{1}$
}

\begin{abstract}
Resumo: Neste artigo, propomos uma ferramenta metodológica para analisar os raciocínios argumentativos expressos por estudantes do ensino médio em um contexto de controvérsia. Coletamos os dados a partir de um questionário que busca investigar como eles utilizam evidências e as relacionam em diferentes justificativas para dar suporte ao ponto de vista que defendem. $\mathrm{Na}$ análise, classificamos os elementos dos argumentos elaborados e identificamos os níveis de complexidade das relações estabelecidas entre os elementos de um argumento, visando analisar o raciocínio argumentativo expresso por eles. Os resultados mostram que a ferramenta é promissora, pois considera os elementos e as relações entre eles presentes no discurso dos sujeitos; contribui para que se entenda como os estudantes constroem seus argumentos para apoiar suas visões; e pode ser usada em diferentes contextos.
\end{abstract}

Palavras-chave: Ensino médio. Raciocínio argumentativo. Metodologia de análise. Argumentação.

\begin{abstract}
In this paper, we propose a methodological tool to analyse the argumentative reasoning expressed by high school students in a controversial context. Data were collected from a questionnaire that aims at investigating how they use evidence and relate them to different warrants in order to support their views. In the analysis, we classify the elements of an argument expressed by students. Then, we identify the complexity levels of the relationships established between elements of an argument in order to analyse students' expressed argumentative reasoning. The results show that the tool is promising, since it considers the elements and the relations between them that compose a given argument; contributes to the understanding of how students build their arguments to support their views; and it may be used in different contexts.
\end{abstract}

Keywords: High school. Argumentative reasoning. Methodology of analysis. Argumentation.

1 Universidade Federal de Minas Gerais (UFMG), Faculdade de Educação, Belo Horizonte, MG, Brasil. E-mail: <marina.r.martins@hotmail.com>. 


\section{Introdução}

Nos últimos anos, tem crescido o número de estudos que analisam a argumentação como processo que promove a aprendizagem de ciência (DRIVER; NEWTON; OSBORNE, 2000; MENDONÇA; JUSTI, 2013; SASSERON; CARVALHO, 2013; SOUTO; SILVA; MUNFORD, 2011; ZOHAR; NEMET, 2002). De acordo com Jiménez-Aleixandre e Erduran (2008), a argumentação é uma forma de discurso que necessita ser desenvolvida em estudantes e deve ser ensinada explicitamente, a partir de atividades estruturadas adequadamente para que os estudantes tenham oportunidades de discutir, avaliar e debater as questões a eles apresentadas. Além disso, essas autoras apontam que há pelo menos cinco dimensões ou contribuições que essa prática epistêmica pode promover no ensino de ciências:

- no contexto educacional, os professores e alunos não têm acesso aos processos cognitivos uns dos outros. A argumentação pode favorecer professores e pesquisadores a acessar esses processos dos estudantes, uma vez que essa prática contribui para que os raciocínios desenvolvidos pelos indivíduos sejam explicitados (JIMÉNEZ-ALEIXANDRE, 2010; OSBORNE, 2014);

- o desenvolvimento de competências comunicativas e, particularmente, do pensamento crítico. Isto porque essa prática compreende a avaliação de múltiplas visões a partir da coordenação de evidências e da explicitação desses pontos de vista (DRIVER; NEWTON; OSBORNE, 2000; DUSCHL; OSBORNE, 2002; JIMÉNEZ-ALEIXANDRE, 2010; KUHN, 1991; OSBORNE, 2014);

- o desenvolvimento do letramento científico e das capacidades de os estudantes falarem e escreverem cientificamente (JIMÉNEZ-ALEIXANDRE, 2010; JIMÉNEZ-ALEIXANDRE; REIGOSA, 2006; KELLY; REGEV; PROTHERO, 2008; OSBORNE, 2014);

- a enculturação dos estudantes nas práticas da ciência e o desenvolvimento de critérios epistêmicos para avaliar o conhecimento, isto é, para que eles compreendam como a ciência é construída e como seus produtos são disseminados (DUSCHL; OSBORNE, 2002; JIMÉNEZ-ALEIXANDRE, 2010; OSBORNE, 2014; SANDOVAL; WILLWOOD, 2008); e

- o desenvolvimento do raciocínio, particularmente da capacidade de escolher entre teorias ou posições baseadas nos critérios de raciocínio (JIMÉNEZ-ALEIXANDRE, 2010; QUEIROZ; SÁ, 2009). Isso colabora para que os estudantes se tornem mais autônomos em seu processo de aprendizagem (JIMÉNEZ-ALEIXANDRE, 2010). Portanto, a argumentação não deve ser dispensável ou tratada de modo superficial nas aulas de ciências, e sim como um elemento essencial desse ambiente de aprendizagem (DUSCHL, 2008).

Apesar do reconhecimento da importância da inserção de práticas argumentativas no ensino de ciências, na literatura não existe consenso acerca de como os argumentos produzidos pelos estudantes podem/devem ser analisados quando se pretende entender a articulação dos mesmos em seus raciocínios. Isto nos motivou a estudar detalhadamente as ferramentas mais utilizadas atualmente na análise de argumentos de estudantes. A partir daí, decidimos propor 
uma outra ferramenta, que busca contemplar as lacunas existentes na literatura. Neste artigo, apresentamos uma síntese de nossos estudos, descrevemos a ferramenta proposta e apresentamos uma avaliação da mesma a partir de sua utilização em um contexto regular de ensino de Química.

\section{Referencial teórico}

Nas últimas duas décadas, pesquisas têm sido desenvolvidas para criar ferramentas capazes de analisar tanto o desenvolvimento do raciocínio dos estudantes com base em evidências e teorias quanto a natureza dialógica dos discursos em salas de aula (DUSCHL, 2008). Uma das ferramentas mais utilizadas para analisar a estrutura dos argumentos foi a proposta por Toulmin (1958). Ele propôs um modelo, denominado Toulmin's Argument Pattern (TAP), que identifica os elementos centrais e as relações entre esses elementos. Tais elementos são: os dados, evidências às quais recorremos para dar suporte às afirmativas; a conclusão, uma afirmativa cujos méritos se procura estabelecer; e a garantia que estabelece uma conexão entre os dados e a conclusão. Mas, um argumento elaborado a partir desses três elementos seria pouco sofisticado. Um argumento mais aprimorado deveria conter, além deles, o qualificador, que estabelece o grau de confiança entre os dados e a conclusão; o apoio, a justificativa da garantia; e a refutação, que indica em que situação a conclusão não é válida.

Segundo alguns pesquisadores (ERDURAN, 2008; SAMPSON; CLARKE, 2008), há algumas dificuldades para se utilizar o TAP. A primeira está relacionada à distinção entre o que é dado, garantia, apoio ou conclusão durante o processo de análise do argumento. A segunda está relacionada à analise de argumentos curtos (KELLY; DRUKER; CHEN, 1998), o que pode não contribuir para que as linhas de raciocínio desenvolvidas pelo sujeito em um determinado contexto sejam identificadas (IBRAIM; MENDONÇA; JUSTI, 2013).

Considerando as limitações do TAP, alguns pesquisadores propuseram adaptações a ele. Por exemplo, Zohar e Nemet (2002) o modificaram para avaliar a qualidade dos argumentos escritos construídos por estudantes com base na sua estrutura e conteúdo. Para isso, elas definem o que é um argumento, elaboram critérios para distinguir um argumento fraco de um forte e para classificar sentenças como argumento ou não. Como resultado do uso dessa ferramenta modificada, elas concluíram que a mesma é viável para a análise de argumentos em contextos sociocientíficos, uma vez que neles as afirmativas opostas podem ser validadas a partir de múltiplas perspectivas. Porém, no contexto científico, no qual o conteúdo é essencial para produzir um argumento, isto é, para articular e apoiar uma explicação, a ferramenta não é viável. Outra restrição da ferramenta modificada é que ela não inclui uma avaliação de como um estudante leva em conta todas as informações disponíveis ao elaborar um argumento (SAMPSON; CLARKE, 2008).

Sandoval e Millwood (2005) também desenvolveram uma ferramenta metodológica para julgar a qualidade de argumentos científicos elaborados pelos estudantes. Eles utilizam critérios para avaliar os argumentos científicos através de duas dimensões. Uma delas se relaciona à qualidade conceitual dos argumentos, isto é, como os estudantes articulam afirmativas causais dentro de um quadro teórico específico e garantem que essas afirmativas sejam sustentadas por evidências disponíveis. A outra se relaciona à qualidade epistemológica dos argumentos e é mensurada analisando-se como os estudantes utilizam evidências suficientes para dar suporte 
à afirmativa; escrevem uma explicação causal coerente ao fenômeno; e incorporam referências retóricas adequadas quando se referem a evidências. De acordo com Sampson e Clarke (2008), tal ferramenta se concentra apenas no conteúdo das justificativas, sem que haja um foco explícito na estrutura do argumento. Assim, a ferramenta é específica a um contexto, o que implica na necessidade de adaptações para que seja aplicada em outros.

A ferramenta de Kelly e Takao (2002) e Takao e Kelly (2003) foi proposta com o objetivo de analisar argumentos escritos longos e complexos elaborados por estudantes em uma disciplina de Oceanografia. Esses deveriam apoiar suas conclusões teóricas a partir de múltiplas representações de dados. Os argumentos produzidos pelos estudantes geralmente compreendiam várias proposições para apoiar suas conclusões. Esta ferramenta se concentra nos status epistêmicos relacionados a essas proposições e a como elas se conectam para construir o argumento. Para utilizá-la, é preciso identificar as proposições encontradas nas discussões e classificá-las com base em seus níveis epistêmicos, os quais são propostos a partir do contexto. Em geral, eles refletem uma distinção entre a descrição dos dados (nível inferior) e as teorias (nível superior). Após classificar os níveis epistêmicos de cada proposição, busca-se identificar como as proposições estão ligadas entre si e produzir uma representação gráfica da estrutura de um argumento através dessas informações. Esta representação é utilizada para examinar os tipos de proposições que o indivíduo utiliza em sua escrita e como ele as coordena em um argumento.

De acordo com Kelly, Regev e Prothero (2008), Kelly e Takao (2002), Oliveira, Batista e Queiroz (2010), Takao e Kelly (2003) há várias limitações nesta ferramenta. As principais delas se relacionam com a necessidade de avaliar o rigor com que as proposições foram identificadas e com que as conexões entre elas foram estabelecidas. A ausência dessas avaliações dificulta determinar se os alunos compreendem as teorias e/ou quão bem os dados sustentam as conclusões. Além disso, Kelly e Takao (2002) encontraram várias discrepâncias entre as maneiras como eles e o instrutor do curso avaliaram os argumentos. Esta variação também pode ser atribuída à falta de precisão na identificação das proposições e/ou de suas inter-relações, isto é, à subjetividade da análise.

Segundo Sampson e Clarke (2008), a ferramenta metodológica proposta por Kelly e Takao (2002) é importante por se concentrar em partes maiores do discurso fundamentado em uma proposição que pode ser considerada uma justificativa em relação à segunda proposição, mas ser considerada uma afirmativa quando associada a uma terceira proposição. Isto significa uma abordagem diferente de analisar a qualidade da argumentação dos sujeitos somente a partir da análise da elaboração de um argumento simples.

Lawson (2003) argumenta que, na educação em ciências, os educadores precisam concentrar seus esforços em ajudar os estudantes a elaborar argumentos que sejam valorizados e utilizados por cientistas ao invés de se concentrar em suas estruturas. Para isso, ele elaborou uma ferramenta que tem como principal objetivo estabelecer a validade de explicações alternativas a partir de um argumento hipotético-presuntivo. Segundo Lawson (2003), argumentos baseados em raciocínio hipotético-dedutível são mais convincentes do que aqueles que se baseiam em evidências, justificativas e apoio para dar suporte às conclusões, uma vez que podem fornecer evidências que, ao mesmo tempo, sustentem determinada explicação e refutem as alternativas. Esse autor considera que o raciocínio argumentativo que apresenta qualidade é o que envolve testes para validar as explicações ou para refutar explicações alternativas através da articulação de afirmativas e evidências. Para Sampson e Clarke (2008), há algumas desvantagens de se 
utilizar essa ferramenta, uma vez que ela não é aplicável em todos os contextos da ciência e se concentra em apenas um dos elementos do argumento: as justificativas. Além disso, o trabalho de Lawson (2003) sugere que os estudantes podem ter dificuldades em desenvolver argumentos hipotético-presuntivos, pois podem não ter desenvolvido o raciocínio necessário para gerar e testar hipóteses. Portanto, é essencial que se procure compreender como os estudantes desenvolvem suas linhas de raciocínio argumentativo para auxiliá-los em sua argumentação.

Esta diversidade de perspectivas para a análise dos argumentos ou dos raciocínios argumentativos nos fornece algumas informações sobre esses tipos de análise. De acordo com Sampson e Clarke (2008) estudos que se concentram na estrutura do argumento demonstram que estudantes tendem a se preocupar mais em construir argumentos que contenham os elementos essenciais do que em analisar se as informações utilizadas por eles são insuficientes ou inadequadas para dar suporte às suas conclusões. Além disso, eles tendem a dar credibilidade às suas experiências pessoais e inferências, assim como às informações fornecidas por figuras de autoridade (por exemplo, professor e/ou livro didático) quando constroem justificativas (ZOHAR; NEMET, 2002). Mesmo quando os estudantes constroem justificativas adequadas para dar suporte às conclusões, geralmente elas não são suficientes, isto é, não são específicas para a conclusão (SANDOVAL; MLLWOOD, 2005). Por outro lado, quando se analisa os raciocínios expressos pelos estudantes, observa-se que eles apresentam linhas de raciocínio diferentes daquelas utilizadas na ciência para sustentar suas conclusões. Ademais, quando se explora esse aspecto, percebe-se que os estudantes procuram utilizar raciocínios hipotético-dedutivos válidos (LAWSON, 2003); partir de observações concretas para ideias mais abstratas (KELLY; TAKAO, 2002); utilizar referências retóricas para tentar estabelecer a validade de um ponto de vista (SANDOVAL; MILLWOOD, 2005); e conectar o conjunto de ideias de forma coerente, adequada e significativa (KELLY; TAKAO, 2002; TAKAO; KELLY, 2003). Sampson e Clarke (2008) apontam que há poucos estudos e ferramentas que se concentram na análise do raciocínio argumentativo explicitado pelos estudantes. Em nossa revisão da literatura, encontramos apenas duas ferramentas: Kelly e Takao (2002) e Lawson (2003). Além disso, tais autores defendem essa abordagem, uma vez que ela pode contribuir para compreender como estudantes sustentam seus pontos de vistas e possibilita caracterizar as relações explicitadas no discurso produzido pelos estudantes, podendo ser aplicada em diferentes contextos.

Segundo Sampson e Clarke (2008), precisamos de ferramentas metodológicas que nos permitam analisar os padrões gerais de justificativas relacionados tanto ao conteúdo dos argumentos, quanto à estrutura dos mesmos. $\mathrm{O}$ estudo aqui relatado se insere nesse contexto de elaboração de ferramentas metodológicas para o campo da argumentação que buscam preencher as lacunas encontradas nas ferramentas existentes.

\section{Objetivos}

Os objetivos desse artigo são: propor uma ferramenta metodológica, baseada nas de Kelly e Takao (2002) e Takao e Kelly (2003), para analisar os raciocínios argumentativos expressos por estudantes do ensino médio em um contexto controverso; e avaliar a utilização da mesma. Mais especificamente, são discutidas as seguintes questões de pesquisa: "Como avaliar o raciocínio argumentativo expresso por estudantes do ensino médio em um contexto que 
envolve uma situação controversa?" e "Como a ferramenta proposta favorece a compreensão dos raciocínios argumentativos expressos pelos estudantes? Por quê?”

\section{Aspectos metodológicos}

\section{Coleta de dados}

Os dados foram coletados em uma turma de primeiro ano do ensino médio regular matutino, composta por 45 alunos, em uma escola pública estadual, localizada em uma grande cidade do sudeste do Brasil. Os estudantes já haviam participado de várias atividades investigativas, aqui entendidas como processos nos quais eles se envolvem ativamente em sua aprendizagem a partir da elaboração de questões, levantamento de hipóteses, análise de evidências e comunicação de resultados. A professora de Química da turma já atuava como professora da rede pública de ensino há dois anos e estava habituada a utilizar essa abordagem de ensino. Além disso, como ela buscava trabalhar explicitamente com argumentação em suas aulas, os alunos sabiam que um argumento deveria ser constituído de afirmativa, justificativa(s) e evidência(s).

A opção pela turma se deveu à disponibilidade da professora e ao consentimento da escola, da professora e dos alunos para a realização da pesquisa.

O instrumento empregado para a coleta de dados foi um questionário cujas questões visavam favorecer a expressão de argumentos produzidos pelos estudantes. Antes do questionário, é apresentado um texto que aborda vários conflitos e discussões de ordem política, sociológica, ética, moral e ambiental a favor e contra a construção da Usina Hidrelétrica de Belo Monte (UHBM), que se iniciou em 2011. Entretanto, a polêmica ocorre desde 1975. Ao elaborar o texto, tivemos o cuidado de apresentar vários aspectos (por exemplo, social, ambiental, ético, moral) e a mesma quantidade de pontos para apoiar as duas perspectivas: a favor e contra a construção da UHBM. É importante mencionar que o tema abordado no texto não era familiar aos estudantes. De acordo com Sandoval e Willwood (2008) quando o tema é familiar, os estudantes apresentam maior facilidade de falar sobre ele, o que implica em uma maior qualidade do raciocínio argumentativo expresso.

O questionário é constituído por três questões. Todas visam investigar como os estudantes subsidiam um determinado ponto de vista, isto é, se e como eles utilizam evidências e as inter-relacionam em diferentes justificativas para dar suporte ao ponto de vista que defendem. Além disso, as questões 2 e 3 apresentam outros objetivos específicos: uma investiga se os estudantes consideram que outro ponto de vista pode ser válido e a outra investiga se eles conseguem pensar em um argumento que seja contrário ao seu posicionamento inicial. Em outras palavras, essas questões favorecem a refutação e a elaboração de teorias alternativas. Esses aspectos são essenciais para favorecer uma análise mais detalhada do raciocínio argumentativo dos estudantes, uma vez que tais habilidades são utilizadas para fundamentar o raciocínio durante o processo argumentativo (KUHN, 1991).

Neste artigo, apresentamos e analisamos os dados de apenas quatro estudantes. Esses dados foram escolhidos por serem mais ricos (em termos da quantidade de argumentos expressos) e, portanto, propiciam uma análise mais detalhada - algo essencial para discutirmos nossa segunda questão de pesquisa. Os nomes utilizados para identificar os estudantes são fictícios 
e isso foi informado a eles no Termo de Consentimento Livre e Esclarecido que foi assinado por todos concordando com a participação na pesquisa.

\section{Referencial de análise}

O referencial de análise proposto neste artigo foi baseado nas ideias de Jiménez-Aleixandre (2010) e Kelly e Takao (2002) e busca investigar o raciocínio argumentativo expresso por estudantes. Como discutido anteriormente, essa perspectiva de análise é mais ampla do que aquelas focadas apenas na estrutura dos argumentos, pois pode ser aplicada em diferentes contextos e possibilita caracterizar as relações explicitadas pelo sujeito.

Para analisar o raciocínio argumentativo expresso pelos estudantes, inicialmente identificamos todos os argumentos produzidos por eles nas três questões do questionário. Utilizamos o referencial teórico de Jiménez-Aleixandre (2010) para classificarmos o que seriam afirmativas (teoria, ponto de vista, ideia ou opinião que se deseja provar), justificativa (razão ou causa que dá suporte a uma afirmativa e a conecta a evidências) e evidência (observação, fato ou dado que oferece suporte à afirmativa) no discurso elaborado pelos estudantes. Essa classificação foi realizada a partir de como eles expressaram esses elementos em seus enunciados. Por exemplo, se um estudante expressou uma relação causal, esta foi considerada como justificativa mesmo se ele apresentasse a evidência como justificativa. Mas, se o estudante apresentasse as informações sem estabelecer uma relação causal, tais informações foram consideradas como evidências. Nesse sentido, tivemos que ampliar a definição de justificativa proposta por Jiménez-Aleixandre (2010), considerando-a como causa que pode dar suporte a uma afirmativa e estabelecer a conexão explicita entre (i) a evidência e a afirmativa; e (ii) as interpretações deduzidas de dados, isto é, as inferências e a afirmativa. Tais inferências se tornam afirmativas causais quando sustentam uma teoria e podem estar, explicita ou implicitamente, apoiadas por evidências que dão suporte à afirmativa. Isso foi feito pois, durante a análise dos dados, percebemos que o suporte às ideias dos estudantes foi feito a partir de justificativas retiradas do texto e de interpretações deduzidas de dados (inferências).

A partir da definição de cada elemento do argumento, classificamos os níveis de complexidade (NC) de cada linha de raciocínio argumentativa (LRA) expressa pelos estudantes (Quadro 1). Consideramos as justificativas que são apoiadas por evidências como sendo de um NC maior do que aquelas em que a evidência não foi explicitada, uma vez que, além de elaborar uma justificativa, elas exigem do estudante selecionar dados relevantes que deem suporte a ela. Portanto, o estabelecimento dessa relação requer do estudante um esforço cognitivo maior.

Avaliamos as evidências (isto é, observações ou fatos que o estudante seleciona no texto para apoiar suas ideias) com NC menor do que as justificativas que não foram apoiadas por evidências. Isto porque estas são proposições que contemplam interpretações deduzidas de dados. Esse mesmo raciocínio foi utilizado quando os estudantes inter-relacionaram: múltiplas evidências; múltiplas justificativas não apoiadas explicitamente por evidências; ou múltiplas justificativas sustentadas por evidências.

Classificamos com o NC 1, a LRA expressa pelo estudante que contemplasse uma ou múltiplas evidências. Em ambos os casos, a relação estabelecida é a mesma, isto é, a evidência sustenta a afirmativa. No entanto, consideramos que a apresentação e o inter-relacionamento de múltiplas evidências apresenta um NC (1B) maior do que a seleção de uma evidência (NC 
1A), uma vez que requer do estudante selecionar vários dados e os inter-relacionar de modo a dar suporte à sua ideia. O mesmo raciocínio foi estabelecido para as justificativas sustentadas, explicitamente ou não, por evidências. Finalmente, em uma LRA que é constituída apenas por evidências, não é possível ter evidências isoladas. Quando isto acontece, cada evidência contempla uma LRA diferente. O mesmo ocorre para as justificativas sustentadas, explicitamente ou não, por evidências. O Quadro 1 apresenta a classificação e descrição dos NC dos relacionamentos.

Quadro 1. Classificação e a descrição dos níveis de complexidade dos relacionamentos

\begin{tabular}{|c|l|}
\hline NC & \multicolumn{1}{|c|}{ Descrição } \\
\hline 1A & Seleção de um dado relevante (evidência) em um conjunto de dados para dar suporte à uma teoria. \\
\hline 1B & $\begin{array}{l}\text { Seleção e inter-relacionamento de múltiplos dados relevantes (evidências) em um conjunto de } \\
\text { dados para dar suporte à uma teoria. }\end{array}$ \\
\hline 2A & Elaboração de uma justificativa não apoiada por evidência para dar suporte à uma teoria. \\
\hline 2B & $\begin{array}{l}\text { Elaboração de múltiplas justificativas inter-relacionadas, mas não apoiadas por evidências, para dar } \\
\text { suporte à uma teoria. }\end{array}$ \\
\hline 3A & Elaboração de uma justificativa apoiada por evidências para dar suporte à uma teoria. \\
\hline 3B & $\begin{array}{l}\text { Elaboração de múltiplas justificativas inter-relacionadas e apoiadas por evidências para dar suporte } \\
\text { à uma teoria. }\end{array}$ \\
\hline
\end{tabular}

Fonte: elaborado pelas autoras

No referencial teórico proposto por Kelly e Takao (2002), a justificativa não está explicitada e não há consideração de afirmativas causais que envolvam o emprego de raciocínio dedutivo. Consideramos importante que tais elementos façam parte de nossa análise, uma vez que são essenciais e bastante utilizadas em processos argumentativos (WALTON; REED; MACAGNO, 2008). Além disso, os níveis epistêmicos que Kelly e Takao elaboraram são específicos para o contexto em que a pesquisa deles se realizou, dificultando a utilização desse referencial em contextos distintos. Por esse motivo, propusemos níveis de complexidade que podem ser utilizados independentemente do contexto, contribuindo para uma análise mais ampla.

Após a classificação dos NC dos relacionamentos, elaboramos uma representação da estrutura dos argumentos que os estudantes expressaram, isto é, buscamos compreender como eles organizaram suas ideias (conectaram suas justificativas e evidências) para apoiar suas teorias. Tal representação (Figura 1) é mais detalhada do que aquela proposta por Kelly e Takao (2002), uma vez que ela explícita a justificativa e considera que a inferência elaborada a partir de um raciocínio dedutivo pode desempenhar o papel de justificativa quando se conecta à uma teoria.

$\mathrm{Na}$ Figura 1, assim como em todas as seguintes:

- Afirmativas são representadas em triângulos.

- Evidências são representadas em elipses. As elipses com fundo cinza apresentam evidências elaboradas pelos estudantes, enquanto as de fundo branco apresentam evidências que foram retiradas do texto. 
- Justificativas são representadas em retângulos. Esses, quando contêm fundo cinza, contém justificativas elaboradas pelos estudantes. A ausência dessa cor indica que as justificativas foram retiradas do texto.

- LRA são compreendidas como formas diferentes de raciocínios para sustentar uma mesma teoria. Portanto, uma teoria pode ser sustentada por várias LRA.

- A LRA que articula mais evidências e justificativas para apoiar uma teoria (por exemplo, a LRA 1, na Figura 1) é considerada mais elaborada, uma vez que os NC das relações estabelecidas são maiores e, portanto, sustentam melhor a teoria do que aquela que articula apenas uma evidência (por exemplo, a LRA 3, na Figura 1).

Figura 1. Representação simplificada da estrutura do argumento



Linha de Raciocínio 1

Linha de Raciocínio 2

Linha de Raciocínio 3

Fonte: elaborada pelas autoras

\section{Análise de dados}

Para sintetizar os dados analisados dos estudantes Geraldo, Isabela, Morgana e Fernando, identificamos, a partir de suas respostas, os esquemas de raciocínios argumentativos desenvolvidos por eles e os representamos em figuras similares à Figura 1. Para facilitar a compreensão do leitor e a discussão dos resultados, optamos por utilizar alguns códigos. Para identificar as teorias, utilizamos os códigos $\mathrm{T} 1_{\mathrm{A} 1}$ e $\mathrm{T} 2{ }_{\mathrm{A} 1}$. A letra T significa Teoria e o número que a acompanha diferencia uma teoria da outra. O índice é composto pela letra $A$, que significa que é uma afirmativa e o número que se segue indica a ordem em que as afirmativas foram expressas. As justificativas estão identificadas pela letra J e o número que se segue indica a ordem em que as mesmas foram expressas. No índice, identificamos à qual teoria cada justificativa se relaciona, isto é, T1 ou T2, e a letra inicial de quem as elaborou. Por exemplo, o código $\mathrm{J} 2_{\mathrm{T} 1 \mathrm{G}}$ significa 
que esta é a segunda justificativa elaborada pelo estudante Geraldo para dar suporte à teoria 1. Os mesmos códigos, mas com utilização da letra inicial $\mathrm{E}$ foram utilizados para as evidências.

Em seguida, construímos os quadros 2 a 5, apresentando os NC desenvolvidos pelos estudantes em cada linha de raciocínio e a descrição das relações dos elementos dos argumentos. O resultado dessa análise foi validado através de triangulação entre juízes em um grupo de 11 pesquisadores da área de Educação em Ciências.

\section{Resultados e discussão}

Visando favorecer a discussão de nossas questões de pesquisa, apresentamos e discutimos nossos resultados em etapas. Inicialmente, focamos nos raciocínios argumentativos de cada aluno separadamente. Em seguida, estabelecemos relacionamentos entre todos os resultados.

\section{Estudante Geraldo}

Como evidenciado na Figura 2, para apoiar a teoria T1 ${ }_{\mathrm{A} 1}$ "A favor da construção da

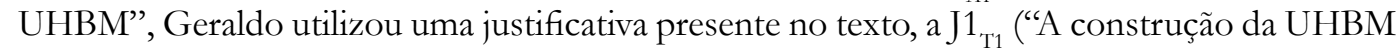
ajudará o Brasil no futuro”). Esta foi sustentada por duas evidências E1 $1_{\mathrm{T} 1}$ e E2 ${ }_{\mathrm{T} 1}$, que foram inter-relacionadas. Além disso, o estudante elaborou a inferência $\mathrm{J} 2_{\mathrm{T} 1}$, que desempenhou o papel de justificativa, uma vez que conectou as evidências $\mathrm{E} 3_{\mathrm{T} 1}$ e $\mathrm{E} 4_{\mathrm{T} 1}$ à justificativa $\mathrm{J} 1_{\mathrm{T} 1}$. Apesar de $\mathrm{J} 1_{\mathrm{T} 1}$ e $2_{\mathrm{T} 1}$ serem justificativas, consideramos que, por ter sido elaborada pelo sujeito, $\mathrm{J} 2_{\mathrm{T} 1}$ exigiu dele um nível cognitivo maior do que $\mathrm{J}_{\mathrm{T} 1}$, que foi selecionada no texto. $\mathrm{O}$ mesmo raciocínio pode ser estabelecido para as evidências. $\mathrm{E} 3_{\mathrm{T} 1}$ exigiu do estudante um nível cognitivo maior do que as demais, uma vez que ela foi criada pelo sujeito, enquanto as outras foram selecionadas no texto. Classificamos que o NC envolvido nessas relações foi 3B, isto é, o estudante elaborou múltiplas justificativas inter-relacionadas e apoiadas por evidências para dar suporte à teoria. Portanto, é possível afirmar que a LRA 1 explicitada pelo estudante é capaz de sustentar a teoria $\mathrm{T} 1_{\mathrm{A} 1}$, uma vez que é composta por afirmativa sustentada por evidências que foram conectadas a partir de justificativas.

Para apoiar a posição contrária, T2 ${ }_{\mathrm{A} 1}$, Geraldo expressou duas LRA (Figura 3). Na primeira, ele elaborou a justificativa $\mathrm{J}_{\mathrm{T} 2}$, sustentada pelas evidências $\mathrm{E} 1_{\mathrm{T} 2}, \mathrm{E} 2_{\mathrm{T} 2}$ e E $3_{\mathrm{T} 2}$. Consideramos que o NC envolvido nessa relação foi $3 \mathrm{~A}$, pois o estudante elaborou uma justificativa apoiada por evidências para dar suporte à teoria. Na outra linha de raciocínio expressa para apoiar $\mathrm{T} 2{ }_{\mathrm{A} 1}$, Geraldo selecionou no texto a justificativa $\mathrm{J} 3_{\mathrm{T} 2}$, que foi sustentada por duas evidências inter-relacionadas: $\mathrm{E} 4_{\mathrm{T} 2}$ e $\mathrm{E} 5_{\mathrm{T} 2}$. Além disso, ele elaborou a justificativa $\mathrm{J} 2_{\mathrm{T} 2}$, que foi conectada às evidências $\mathrm{E} 4_{\mathrm{T} 2}$ e $\mathrm{E} 5_{\mathrm{T} 2}$ através da justificativa $3_{\mathrm{T} 2}$. Consideramos que $\mathrm{J} 2_{\mathrm{T} 2}$ exigiu de Geraldo um nível cognitivo maior do que $\mathrm{J}_{\mathrm{T} 2}$, uma vez que essa envolveu apenas a seleção de justificativas presentes no texto, enquanto aquela envolveu uma interpretação deduzida das informações contidas no mesmo. Como o estudante elaborou múltiplas justificativas inter-relacionadas e apoiadas por evidências para dar suporte à teoria, avaliamos o NC dessas relações como 3B. 
Comparando os raciocínios argumentativos expressos por Geraldo para dar suporte à $\mathrm{T} 1_{\mathrm{A} 1}$ e $\mathrm{T} 2_{\mathrm{Al}}$, concluímos que o estudante deu maior suporte à teoria $\mathrm{T} 2_{\mathrm{AI}}$, uma vez que desenvolveu uma LRA a mais em relação à T2 ${ }_{\text {AI }}$ (ver figuras 2 e 3 e Quadro 2).

Figura 2. Esquema de raciocínio argumentativo elaborado por Geraldo a favor da construção da UHBM

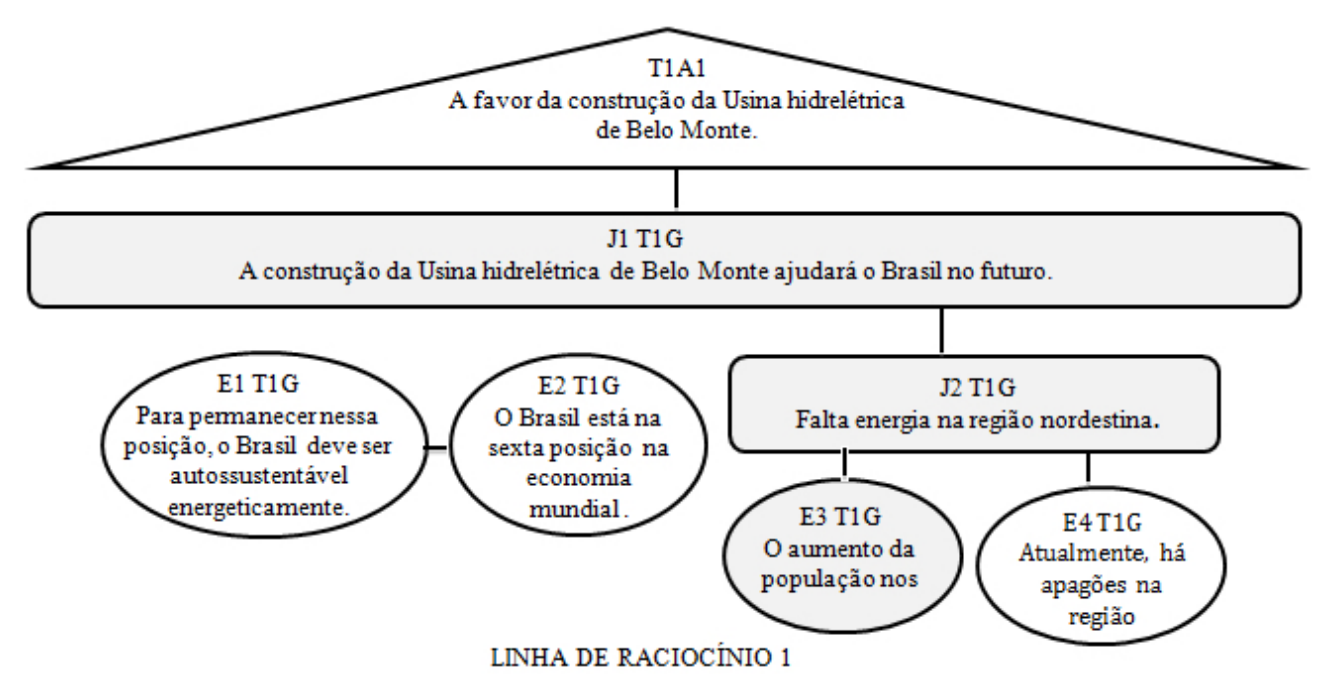

Fonte: elaborada pelas autoras.

Figura 3. Esquema de raciocínio argumentativo elaborado por Geraldo contra a construção da UHBM

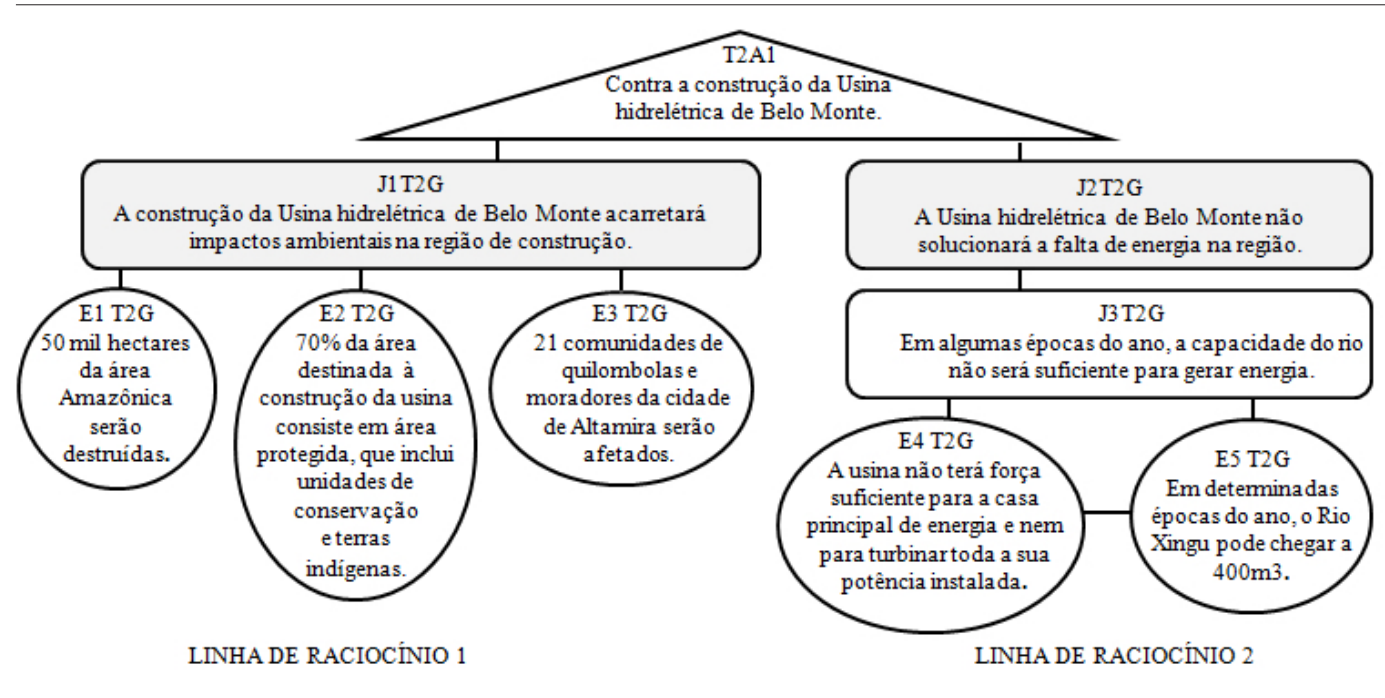

Fonte: elaborada pelas autoras. 
Quadro 2. Linhas de raciocínio, níveis de complexidade e descrição das relações dos elementos dos argumentos desenvolvidos por Geraldo

\begin{tabular}{|c|l|l|l|}
\hline Posição & LRA & NC & \multicolumn{1}{|c|}{ Descrição } \\
\hline $\begin{array}{c}\text { A favor da } \\
\text { construção da } \\
\text { UHBM }\end{array}$ & 1 & $3 \mathrm{~B}$ & $\begin{array}{l}\text { Elaboração de múltiplas justificativas inter-relacionadas e } \\
\text { apoiadas por evidências para dar suporte à teoria. }\end{array}$ \\
\hline $\begin{array}{c}\text { Contra a } \\
\text { construção da } \\
\text { UHBM }\end{array}$ & 1 & $3 \mathrm{~A}$ & $\begin{array}{l}\text { Elaboração de uma justificativa apoiada por evidências para } \\
\text { dar suporte à teoria. }\end{array}$ \\
\cline { 2 - 5 } & 2 & $3 \mathrm{~B}$ & $\begin{array}{l}\text { Elaboração de múltiplas justificativas inter-relacionadas e } \\
\text { apoiadas por evidências para dar suporte à teoria. }\end{array}$ \\
\hline
\end{tabular}

Fonte: elaborado pelas autoras.

\section{Estudante Isabela}

A estudante Isabela expressou três LRA para dar suporte à teoria $\mathrm{T} 1_{\mathrm{A} 1}$ (Figura 4). $\mathrm{Na}$ primeira, ela elaborou $\mathrm{J}_{\mathrm{T} 1 \mathrm{I}}$ para oferecer suporte à afirmativa $\mathrm{T} 1_{\mathrm{A} 1}$. Consideramos o $\mathrm{NC}$ dessa relação como 2A. Portanto, nessa linha de raciocínio, Isabela não deu suporte à justificativa através de evidências. A terceira LRA é formada por apenas uma evidência cujo relacionamento com a teoria foi explícito, o que resultou em sua classificação como NC 1A. A segunda LRA é a mais desenvolvida das três, uma vez que Isabela desenvolveu a justificativa $\mathrm{J}_{\mathrm{T} 1 \mathrm{I}}$ apoiada por uma evidência, $\mathrm{E} 1_{\mathrm{T} 1}$, também criada por ela para dar suporte à $\mathrm{T} 1_{\mathrm{A} 1}$. Assim, consideramos $\mathrm{O}$ $\mathrm{NC}$ dessa relação como $3 \mathrm{~A}$.

A estudante também expressou linhas de raciocínio argumentativas para apoiar a teoria contrária, T2 $2_{\mathrm{A} 1}$ (Figura 5). Na primeira delas, ela elaborou as justificativas $\mathrm{J}_{\mathrm{T} 2 \mathrm{I}}$ e J2 ${ }_{\mathrm{T} 2 \mathrm{I}}$ e as inter-relacionou para dar suporte à teoria $\mathrm{T} 2 \mathrm{~A}$. Por isso avaliamos o $\mathrm{NC}$ dessa relação como 2B. Na segunda e terceira LRA, ela criou as evidências E1 ${ }_{\mathrm{T} 2 \mathrm{I}}$ e E2 ${ }_{\mathrm{T} 2 \mathrm{I}}$ para dar suporte à teoria $\mathrm{T} 2{ }_{\mathrm{A} 1}$. Assim, classificamos os NC dessas relações como $1 \mathrm{~A}$.

Comparando as linhas de raciocínio argumentativo expressas pela estudante para defender as teorias $\mathrm{T}_{\mathrm{A} 1}$ e $\mathrm{T} 2{ }_{\mathrm{A} 1}$, observamos que ela expressou a mesma quantidade de LRA para dar suporte a cada teoria. No entanto, os NC das relações expressas para dar suporte à $\mathrm{T} 1_{\mathrm{A} 1}$ são maiores do que aqueles usados para dar suporte à $\mathrm{T} 2_{\mathrm{A} 1}$. Isto significa que algumas das LRA usadas para dar suporte à $\mathrm{T} 1_{\mathrm{A} 1}$ foram mais bem desenvolvidas do que as que deram apoio à $\mathrm{T} 2_{\mathrm{A} 1}$ (ver figuras 4 e 5 e Quadro 3). 
Figura 4. Esquema de raciocínio argumentativo elaborado por Isabela a favor da construção da UHBM



Fonte: elaborada pelas autoras.

Figura 5. Esquema de raciocínio argumentativo elaborado por Isabela contra a construção da UHBM

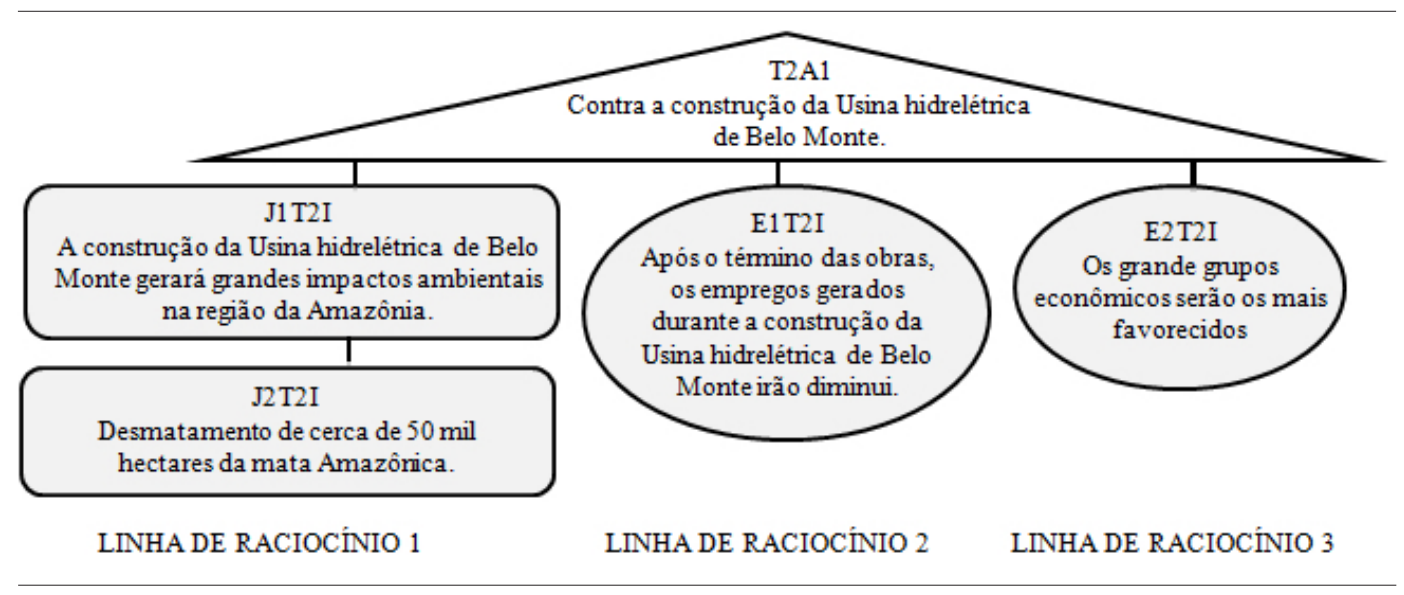

Fonte: elaborada pelas autoras. 
Quadro 3. Linhas de raciocínio, níveis epistêmicos e descrição das relações dos elementos dos argumentos desenvolvidos por Isabela

\begin{tabular}{|c|c|c|c|}
\hline Posição & LRA & NC & Descrição \\
\hline \multirow{3}{*}{$\begin{array}{l}\text { A favor da } \\
\text { construção da } \\
\text { UHBM }\end{array}$} & 1 & $2 \mathrm{~A}$ & $\begin{array}{l}\text { Elaboração de uma justificativa não apoiada por evidências para } \\
\text { dar suporte à teoria. }\end{array}$ \\
\hline & 2 & $3 \mathrm{~A}$ & $\begin{array}{l}\text { Elaboração de uma justificativa apoiada por evidências para dar } \\
\text { suporte à teoria. }\end{array}$ \\
\hline & 3 & $1 \mathrm{~A}$ & $\begin{array}{l}\text { Seleção de um dado relevante dentro de um conjunto de dados } \\
\text { para dar suporte à teoria. }\end{array}$ \\
\hline \multirow{3}{*}{$\begin{array}{c}\text { Contra a } \\
\text { construção da } \\
\text { UHBM }\end{array}$} & 1 & $2 \mathrm{~B}$ & $\begin{array}{l}\text { Elaboração de múltiplas justificativas inter-relacionadas, mas } \\
\text { não apoiadas por evidências para dar suporte à teoria. }\end{array}$ \\
\hline & 2 & $1 \mathrm{~A}$ & $\begin{array}{l}\text { Seleção de um dado relevante dentro de um conjunto de dados } \\
\text { para dar suporte à sua teoria. }\end{array}$ \\
\hline & 3 & $1 \mathrm{~A}$ & $\begin{array}{l}\text { Seleção de um dado relevante dentro de um conjunto de dados } \\
\text { para dar suporte à sua teoria }\end{array}$ \\
\hline
\end{tabular}

Fonte: elaborado pelas autoras.

\section{Estudante Morgana}

Para apoiar a teoria $\mathrm{T}_{\mathrm{A} 1}$, Morgana elaborou uma LRA na qual utilizou duas evidências, $\mathrm{E} 1_{\mathrm{T} 1 \mathrm{M}}$ e E2 $2_{\mathrm{T} 1 \mathrm{M}}$, que foram conectadas à $\mathrm{T} 1_{\mathrm{A1}}$ através da justificativa $\mathrm{J} 1_{\mathrm{T} 1 \mathrm{M}}$ (Figura 6). Classificamos o NC dessa relação como $3 \mathrm{~A}$, uma vez que a estudante elaborou uma justificativa apoiada por evidências para dar suporte à teoria.

Figura 6. Esquema de raciocínio argumentativo elaborado por Morgana a favor da construção da UHBM
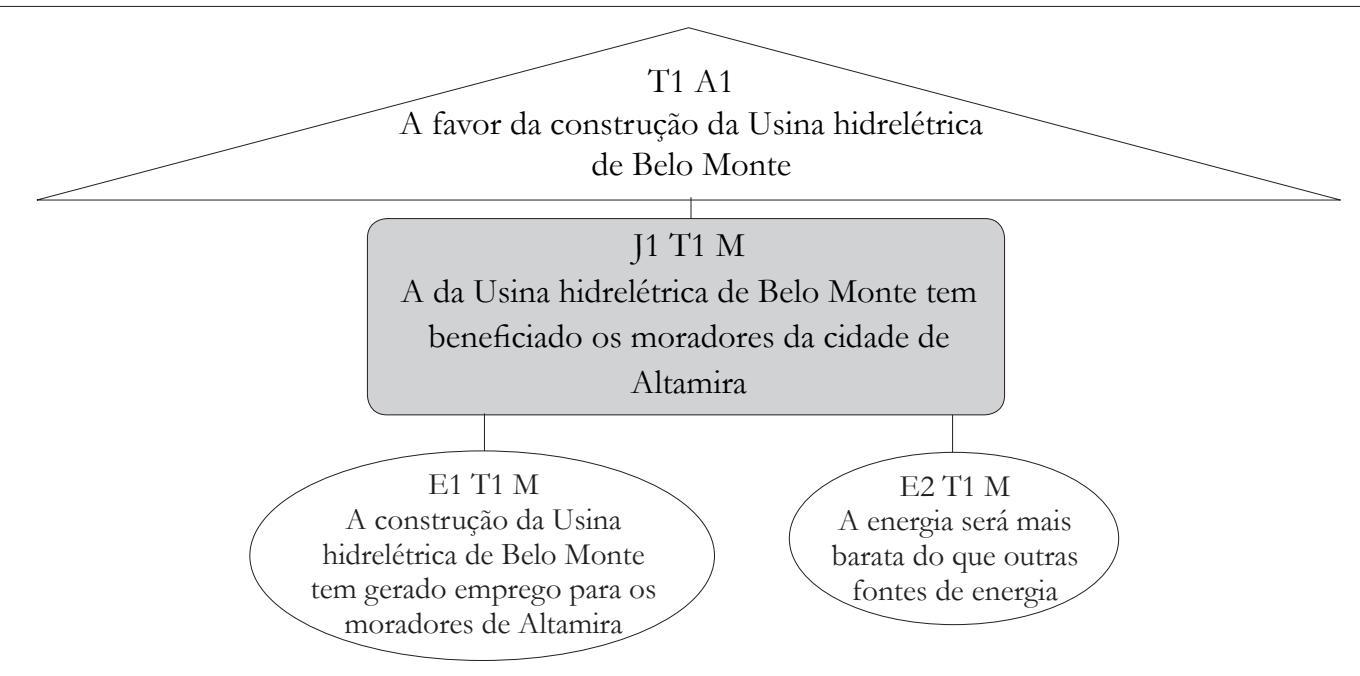

\section{LINHA DE RACIOCÍNIO 1}

Fonte: elaborada pelas autoras. 
Para sustentar a teoria T2 ${ }_{\mathrm{A} 1}$, Morgana expressou quatro LRA (Figura 7). A primeira, a segunda e a quarta são compostas por uma evidência cada, sendo que as evidências que compõem as linhas 1 e 4 foram criadas pela estudante. Então, elas foram classificadas com o mesmo NC: $1 \mathrm{~A}$. Na terceira LRA, a estudante elaborou uma justificativa, $\mathrm{J}_{\mathrm{T} 2 \mathrm{M}}$, não apoiada por evidências, para dar suporte à teoria. Por isso consideramos que essa relação apresenta NC 2A sendo, portanto, a mais sofisticada das quatro LRA expressas por Morgana.

Figura 7. Esquema de raciocínio argumentativo elaborado por Morgana contra a construção da UHBM



LINHA DE RACIOCÍNIO 1

LINHA DE RACIOCÍNIO 2

LINHA DE RACIOCÍNIO 3

LINHA DE RACIOCÍNIO 4

Fonte: elaborada pelas autoras.

Comparando os raciocínios argumentativos expressos pela estudante para apoiar as duas teorias, constatamos que $\mathrm{T} 1_{\mathrm{A} 1}$ foi mais sustentada do que $\mathrm{T} 2_{\mathrm{A} 1}$. Apesar de Morgana expressar um maior número de LRA para apoiar $\mathrm{T} 2{ }_{\mathrm{A} 1}$, essas foram pouco desenvolvidas (apresentação de três evidências e uma justificativa em diferentes linhas) em comparação à LRA que deu suporte à $\mathrm{T} 1_{\mathrm{A} 1}$, composta por uma justificativa apoiada por duas evidências (ver figuras 6 e 7 e Quadro 4).

Quadro 4. Linhas de raciocínio, níveis de complexidade e descrição das relações dos elementos dos argumentos desenvolvidos por Morgana

\begin{tabular}{|c|l|l|l|}
\hline Posição & LRA & NC & \multicolumn{1}{|c|}{ Descrição } \\
\hline $\begin{array}{c}\text { A favor da construção } \\
\text { da UHBM }\end{array}$ & 1 & $3 \mathrm{~A}$ & $\begin{array}{l}\text { Elaboração de uma justificativa apoiada por evidências para } \\
\text { dar suporte à teoria. }\end{array}$ \\
\hline \multirow{2}{*}{$\begin{array}{c}\text { Contra a construção } \\
\text { da UHBM }\end{array}$} & 1 & $1 \mathrm{~A}$ & $\begin{array}{l}\text { Seleção de um dado relevante dentro de um conjunto de } \\
\text { dados para dar suporte à teoria. }\end{array}$ \\
\cline { 2 - 4 } & 2 & $1 \mathrm{~A}$ & $\begin{array}{l}\text { Seleção de um dado relevante dentro de um conjunto de } \\
\text { dados para dar suporte à teoria. }\end{array}$ \\
\cline { 2 - 4 } & $2 \mathrm{~A}$ & $\begin{array}{l}\text { Elaboração de uma justificativa não apoiada por evidências } \\
\text { para dar suporte à teoria. }\end{array}$ \\
\cline { 2 - 4 } & 4 & $1 \mathrm{~A}$ & $\begin{array}{l}\text { Seleção de um dado relevante dentro de um conjunto de } \\
\text { dados para dar suporte à teoria. }\end{array}$ \\
\hline
\end{tabular}

Fonte: elaborado pelas autoras. 


\section{Estudante Fernando}

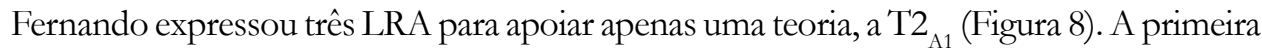
é sustentada por uma evidência criada por ele: $\mathrm{E} 1_{\mathrm{T} 2 \mathrm{~F}}$ Assim, classificamos o $\mathrm{NC}$ dessa relação como 1A. A segunda e a terceira LRA são compostas por uma justificativa cada. Apesar de as classificamos com o mesmo NC, 2A, consideramos que o nível cognitivo da $\mathrm{J}_{\mathrm{T} 2 \mathrm{~F}}$ é maior do que a da J2 ${ }_{\mathrm{T} 2 \mathrm{~F}} \mathrm{em}$ virtude daquela ter sido elaborada pelo estudante e a $2_{\text {T2F }}$ selecionado do texto. Consideramos que essas LRA exibiram um NC maior do que a primeira, que é constituída por apenas uma evidência (NC 1) (ver Figura 8 e Quadro 5).

Figura 8. Esquema de raciocínio argumentativo elaborado por Fernando contra a construção da UHBM

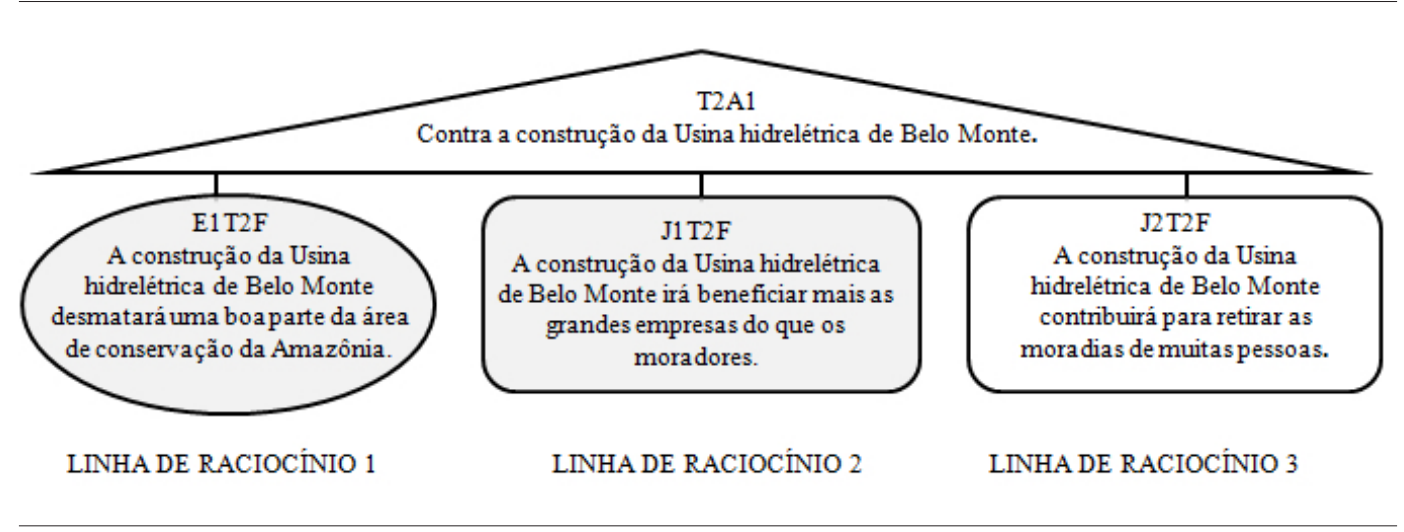

Fonte: elaborada pelas autoras.

Quadro 5. Linhas de raciocínio, níveis de qualidade e descrição das relações dos elementos dos argumentos desenvolvidos por Fernando

\begin{tabular}{|c|l|l|l|}
\hline Posição & LRA & NC & \multicolumn{1}{|c|}{ Descrição } \\
\hline \multirow{2}{*}{$\begin{array}{c}\text { Contra a construção } \\
\text { da UHBM }\end{array}$} & 1 & $1 \mathrm{~A}$ & $\begin{array}{l}\text { Seleção de um dado relevante dentro de um conjunto de } \\
\text { dados para dar suporte à sua teoria. }\end{array}$ \\
\cline { 2 - 4 } & 2 & 2A & $\begin{array}{l}\text { Elaboração de uma justificativa não apoiada por evidência } \\
\text { para dar suporte à teoria. }\end{array}$ \\
\cline { 2 - 4 } & 3 & 2A & $\begin{array}{l}\text { Elaboração de uma justificativa não apoiada por evidência para dar } \\
\text { suporte à teoria. }\end{array}$ \\
\hline
\end{tabular}

Fonte: elaborado pelas autoras. 


\section{Comparando os raciocínios argumentativos expressos pelos estudantes}

A partir do que foi discutido para cada estudante, podemos concluir que Geraldo foi o sujeito que expressou o raciocínio argumentativo mais complexo, uma vez que ele elaborou múltiplas justificativas inter-relacionadas e apoiadas por evidências para dar suporte às teorias, principalmente $\mathrm{T} 2{ }_{\mathrm{A} 1}$ (ver figuras 2 e 3 e Quadro 2).

Por outro lado, Fernando foi o que apresentou o raciocínio argumentativo mais simples em relação aos outros, isto é, com NC das relações menos elevados. Apesar de apresentar três linhas de raciocínio argumentativas, elas apresentavam elementos e relações simples entre eles (ver Figura 8 e Quadro 5). Além disso, Fernando foi o único dos estudantes analisados que não conseguiu dar suporte à uma teoria alternativa.

Entre as estudantes Morgana e Isabela, esta elaborou um suporte melhor às teorias $\mathrm{T} 1_{\mathrm{A} 1}$ e $\mathrm{T} 2_{\mathrm{A} 1}$. Em relação à teoria $\mathrm{T} 1_{\mathrm{A} 1}$, Isabela desenvolveu três LRA, enquanto Morgana desenvolveu apenas uma. Elas expressaram o mesmo $\mathrm{NC}$ em uma dessas linhas (ver figuras 4 e 6 e quadros 3 e 4). Para dar suporte à T2 $2_{\mathrm{A} 1}$, Isabela desenvolveu melhor uma de suas LRA, uma vez que elaborou múltiplas justificativas inter-relacionadas, mas que não foram apoiadas por evidências. Apesar de Morgana ter desenvolvido uma LRA a mais do que Isabela, elas foram pouco desenvolvidas (ver figuras 5 e 7 e quadros 3 e 4).

É importante mencionar que o único estudante que elaborou justificativas apoiadas por evidências para dar suporte às duas teorias foi Geraldo. Todos os outros estudantes tiveram dificuldades de integrar as evidências a alguma teoria elaborando justificativas. Isso nos leva a inferir que eles consideram que os dados falam por si só. Sandoval e Millwood (2005) também encontraram esse mesmo resultado em seu trabalho, isto é, que os estudantes apresentam dificuldades em relacionar os dados com a ideia defendida. Outro aspecto evidenciado a partir da análise das LRA é que, na maioria delas, esses mesmos estudantes não sustentaram suas justificativas a partir de evidências. Possivelmente, isto ocorreu em função de os estudantes considerarem que a elaboração de uma justificativa era suficiente para dar suporte às suas ideias.

A partir do uso dessa ferramenta de análise, constatamos também que apenas Geraldo e Isabela, os estudantes que melhor desenvolveram suas LRA, conseguiram elaborar contra-argumentos, isto é, apontar falhas em suas teorias. Geraldo havia mencionado que era "a favor da construção da UHBM, pois esta ajudaria o Brasil no futuro, uma vez que há falta de energia na região nordestina devido a apagões". Mas, para apoiar a teoria T2 ${ }_{\mathrm{A} 1}$, ele havia dito que "a usina bidrelétrica de Belo Monte não solucionará o problema de falta de energia na região, pois em algumas épocas do ano, a capacidade do rio não será suficiente para gerar energia devido aos fatos de não ter força suficiente para a casa principal de energia e nem para turbinar toda a sua potência, uma vez que em determinadas épocas do ano, o Rio Xingu pode chegar a $400 \mathrm{~m}$ ". Isabela afirmou que "A construção da Usina bidrelétrica de Belo Monte não acarretará grandes impactos ambientais" (A2 $\left.{ }_{\mathrm{T} 11}\right)$ para apoiar $\mathrm{T} 1_{\mathrm{A} 1}$. No entanto, para apoiar $\mathrm{T} 2_{\mathrm{A} 1}$, ela disse que " $A$ construção da usina hidrelétrica de Belo Monte gerará grandes impactos ambientais na região da Amazônia, pois haverá desmatamento de cerca de 50 mil hectares da mata Amazônica". Segundo Kuhn (1991), a habilidade de contra argumentar é complexa, uma vez que os sujeitos que apresentam maior convicção de sua resposta inicial têm maiores dificuldades em contra argumentar e maior facilidade em refutar e vice-versa. 


\section{Conclusões e implicações}

A primeira questão de pesquisa que norteou esse estudo é: “Como avaliar o raciocínio argumentativo expresso por estudantes do ensino médio em um contexto que envolve uma situação controversa?". A ferramenta metodológica proposta neste trabalho caracteriza as relações presentes no discurso explicitado pelo sujeito. Isto atende à crítica discutida anteriormente de que a análise de fragmentos isolados do discurso não contribui para compreender como os sujeitos argumentam, nem a qualidade da argumentação desenvolvida por eles.

Outro aspecto com o qual nos preocupamos foi a estrutura do argumento, isto é, a presença de elementos como: afirmativa, evidências e justificativas - algo que é defendido por vários autores (por exemplo, IBRAIM; MENDONÇA; JUSTI, 2013; KELLY; DRUKER; CHEN, 1998; SAMPSON; CLARKE, 2008). No modelo proposto por Kelly e Takao (2002), a justificativa não está explicitada e não há consideração de afirmativas causais que envolvam o emprego de raciocínio dedutivo. Consideramos esses elementos essenciais, uma vez que são bastante utilizados em processos argumentativos (WALTON; REED; MACAGNO, 2008).

Levamos em consideração também a qualidade da argumentação desenvolvida pelo estudante, algo que tem chamado a atenção de alguns estudiosos como Sampson e Clarke (2008), Kelly e Takao (2002). Nesse sentido, estabelecemos NC diferentes para os relacionamentos entre os elementos de um argumento. Procuramos discutir o raciocínio argumentativo que o sujeito explicitou para apoiar suas teorias a partir da presença desses elementos e da coerência do relacionamento entre eles. Portanto, os NC que desenvolvemos e o referencial teórico de Jiménez-Aleixandre (2010), no qual nos baseamos para definir os elementos de um argumento, nos possibilitaram uma análise ampla dos dados e com pouca subjetividade. Kelly e Takao apontam como uma limitação de se utilizar sua ferramenta o fato de não haver um critério para conectar as proposições e nem para avaliar o rigor dessas (KELLY; TAKAO, 2002; KELLY; REGEV; PROTHERO, 2008; TAKAO; KELLY, 2003). Além disso, como enfatizado anteriormente, os NC propostos na ferramenta desses autores são específicos para um contexto, o que dificulta a utilização desse referencial.

A avaliação dos raciocínios argumentativos expressos pelos estudantes se constituiu em uma dimensão maior de análise de como eles constroem seus argumentos para apoiar suas visões, isto é, sobre se e como eles relacionam os elementos do argumento. Isso favoreceu discutir nossa segunda questão de pesquisa (Como a ferramenta proposta favorece a compreensão dos raciocínios argumentativos expressos pelos sujeitos? Por quê?). Tal discussão nos mostrou também que cada estudante desenvolveu as LRA de maneira diferente. Finalmente, acreditamos que a ferramenta proposta pode ser utilizada em vários contextos de ensino, uma vez que seu foco está nas relações entre os elementos dos argumentos explicitados pelo sujeito. Isto abre novas possibilidades de pesquisa, uma vez que a análise realizada neste trabalho contemplou apenas um contexto. A partir da utilização da ferramenta em outros contextos, poderemos considerar a relevância de apresentar e discutir este referencial para professores de Ciências interessados em entender melhor como os estudantes desenvolvem suas LRA. Vislumbramos que isto possa dar suporte a ações voltadas para favorecer a melhoria da qualidade das LRA de seus alunos. 


\section{Agradecimentos}

As autoras agradecem a colaboração do Conselho Nacional de Desenvolvimento Científico e Tecnológico (CNPq) e da Coordenação de Aperfeiçoamento de Pessoal de Nível Superior (CAPES).

\section{Referências}

DRIVER, R.; NEWTON, P.; OSBORNE, J. Establishing the norms of scientific argumentation in classrooms. Science Education, Hoboken, v. 84, n. 3, p. 287-312, 2000. Disponível em: < https://doi.org/10.1002/(SICI)1098-237X(200005)84:3<287::AID-SCE1>3.0.CO;2-A>. Acesso em: 9 jan. 2017.

DUSCHL, R. Quality argumentation and epistemic criteria. In: ERDURAN. S.; JIMÉNEZALEIXANDRE, M. P. (Ed.). Argumentation in science education: perspectives from classroom-based research. Dordrecht: Springer, 2008. p. 159-178.

DUSCHL, R. A.; OSBORNE, J. Supporting and promoting argumentation discourse in science education. Studies in Science Education, Abingdon, v. 38, n. 1, p. 39-72, 2002. Disponível em: <https://doi.org/10.1080/03057260208560187>. Acesso em: 9 jan. 2017.

ERDURAN, S. Methodological foundations in study of argumentation in science classrooms. In: ERDURAN. S.; JIMÉNEZ-ALEIXANDRE, M. P. (Ed.). Argumentation in science education: perspectives from classroom-based research. Dordrecht: Springer, 2008. p. 47-70.

IBRAIM, S. S.; MENDONÇA, P. C. C.; JUSTI, R. Contribuições dos esquemas argumentativos de Walton para análise de argumentos no contexto do ensino de ciências. Revista Brasileira de Pesquisa em Educação em Ciências, Belo Horizonte, v. 13, n. 1, p. 159-185, 2013. Disponível em: <https://seer.ufmg.br/index.php/rbpec/article/viewFile/2460/1860>. Acesso em: 9 jan. 2017.

JIMÉNEZ-ALEIXANDRE, M. P. 10 ideas clave: competencias en argumentación y uso de pruebas. Barcelona: Graó, 2010.

JIMÉNEZ-ALEIXANDRE, M. P.; ERDURAN, S. Argumentation in science education: an overview. In: ERDURAN. S.; JIMÉNEZ-ALEIXANDRE, M. P. (Ed.). Argumentation in science education: perspectives from classroom-based research. Dordrecht: Springer, 2008. p. 3-27.

JIMÉNEZ-ALEIXANDRE, M. P.; REIGOSA, C. Contextualizing practices across epistemic levels in the chemistry laboratory. Science Education, Hoboken, v. 90, n. 4, p. 707-733, 2006. Disponível em: <https://doi.org/10.1002/sce.20132>. Acesso em: 9 jan. 2017.

KELLY, G.; TAKAO, A. Epistemic levels in argument: an analysis of university oceonagraphy students' use of evidence in writing. Science Education, Hoboken, v. 86, n. 3, p. 314-342, 2002. Disponível em: <https://doi.org/10.1002/sce.10024>. Acesso em: 9 jan. 2017. 
KELLY, G. J.; DRUKER, S.; CHEN, C. Student's reasoning about electricity: combining performance assessments with argumentation analysis. International Journal of Science Education, Abingdon, v. 20, n. 7, p. 849-871, 1998. Disponível em: <https://doi. org/10.1080/0950069980200707>. Acesso em: 9 jan. 2017.

KELLY, G. J.; REGEV, J.; PROTHERO, W. Analysis of lines of reasoning in written argumentation. In: ERDURAN. S.; JIMÉNEZ-ALEIXANDRE, M. P. (Ed.). Argumentation in science education: perspectives from classroom-based research. Dordrecht: Springer, 2008. p. $137-158$.

KUHN, D. The skills of argument. New York: Cambridge University, 1991.

LAWSON, A. E. The nature and development of hypothetico-predictive argumentation with implications for science teaching. International Journal of Science Education, Abingdon, v. 25, n. 11, p.1387-1408, 2003. Disponível em: <https://doi.org/10.1080/095006903200005211 7>. Acesso em: 9 jan. 2017.

MENDONÇA, P. C. C.; JUSTI, R. The relationships between modelling and argumentation from the perspective of the model of modelling diagram. International Journal of Science Education, Abingdon, v. 35, n. 14, p. 2007-2034, 2013. Disponível em: < https://doi.org/10.108 0/09500693.2013.811615>. Acesso em: 9 jan. 2017.

OLIVEIRA, J. R. S.; BATISTA, A. A.; QUEIROZ, S. L. Escrita científica de alunos de graduação em química: análise de relatórios de laboratório. Química Nova, São Paulo, v. 33, n. 9, p. 1980-1986, 2010. Disponível em: <https://doi.org/10.1590/S0100-40422010000 900029>. Acesso em: 9 jan. 2017.

OSBORNE, J. Teaching scientific practices: meeting the challenge of change. Journal of Science Teacher Education, Dordrecht, v. 25, n. 2, p. 177-196, 2014. Disponível em: <https:// doi.org/10.1007/s10972-014-9384-1>. Acesso em: 9 jan. 2017.

QUEIROZ, S. L.; SÁ, L. P. O espaço para a argumentação no ensino superior de química. Educación Química, México, v. 20, n. 2, p. 104-110, 2009.

SAMPSON, V.; CLARKE, D. Assessment of the ways students generate arguments in science education: current perspectives and recommendations for future directions. Science Education, Hoboken, v. 92, n. 3, p. 447-472, 2008. Disponível em: <https://doi.org/10.1002/sce.20276>. Acesso em: 9 jan. 2017.

SANDOVAL, W. A.; MILLWOOD, K. A. The quality of students' use of evidence in written scientific explanations. Cognition and Instruction, Philadelphia, v. 23, n. 1, p. 23-55, 2005. Disponível em: <https://doi.org/10.1207/s1532690xci2301_2>. Acesso em: 9 jan. 2017.

What can argumentation tell us about epistemology? In: ERDURAN. S.; JIMÉNEZALEIXANDRE, M. P. (Ed.). Argumentation in science education: perspectives from classroom-based research. Dordrecht: Springer, 2008. p. 71-88. 
SASSERON, L. H.; CARVALHO, A. M. P. Ações e indicadores da construção do argumento em aula de ciências. Ensaio: pesquisa em educação em ciências, Belo Horizonte, v. 15, n. 2 , p. 169-189, 2013. Disponível em: < http://www.portal.fae.ufmg.br/seer/index.php/ensaio/ article/view/844>. Acesso em: 9 jan. 2017.

SOUTO, A. P. S.; SILVA, D. O.; MUNFORD, D. Argumentação no ensino de conceitos da biologia: práticas de um professor de ciências em salas de aula da educação de jovens e adultos. In: ENCONTRO NACIONAL DE EDUCAÇÃO EM CIÊNCIAS, 8., 2011, Campinas. Atas... Campinas: ABRAPEC, 2011.

TAKAO, A. Y.; KELLY, G. J. Assessment of evidence in university students' scientific writing.Science \& Education, Dordrecht, v. 12, n. 4, p. 341-363, 2003. Disponível em: <https://doi. org/10.1023/A:1024450509847>. Acesso em: 9 jan. 2017.

TOULMIN, S. The uses of argument. New York: Cambridge University Press, 1958.

WALTON, D. N.; REED, C.; MACAGNO, F. Argumentation schemes. Cambridge: Cambridge University Press, 2008.

ZOHAR, A.; NEMET, F. Fostering students' knowledge and argumentation skills through dilemmas in human genetics. Journal of Research in Science Teaching, Hoboken, v. 39, n. 1, p. 35-62, 2002. Disponível em: < https://doi.org/10.1002/tea.10008>. Acesso em: 9 jan. 2017.

Artigo recebido em 08/10/2015. Aceito em 14/05/2016.

Endereço para contato: Universidade Federal de Minas Gerais (UFMG), Avenida Antônio Carlos, 6627, Pampulha, Belo Horizonte, MG, CEP 31270-901, Brasil. 